\title{
Diagnóstico por el laboratorio de las enfermedades periodontales y periimplantarias. Diagnóstico de la periodontitis
}

\author{
BULLÓN FERNÁNDEZ P*
}

\begin{abstract}
Bullón Fernández P. Diagnóstico por el laboratorio de las enfermedades periodontales y periimplantarias. Diagnóstico de la periodontitis. Av Periodon Implantol. 2004; 16, 1:35-45.
\end{abstract}

\begin{abstract}
RESUMEN
Desde el punto de vista epidemiológico la enfermedad periodontal tiene una enorme trascendencia. El diagnóstico del proceso es una necesidad para, con arreglo a él, realizar el tratamiento y la prevención mas apropiada. El enfoque del diagnóstico depende del concepto que sobre la patogenia se tiene en un momento dado. Desde esa perspectiva se hace un análisis de los distintos métodos de diagnóstico que existen. Se llega a la conclusión de que actualmente el método clínico, mediante el sondaje periodontal, es el mas apropiado para conocer el estado de gravedad del proceso y enfocar el tratamiento. Tras la revisión de los distintos procesos que estudian: los patógenos responsables, los mediadores de la inflamación, los parámetros de destrucción ósea y del conectivo, los factores genéticos y de riesgo ambiental y adquirido, se llega a la conclusión de que actualmente no existe ningún método capaz de identificar a la población de riesgo de padecer la enfermedad periodontal ni los períodos de actividad. Ello en gran medida se debe a que existen muchas incógnitas en la patogenia del proceso y que es preciso seguir la búsqueda de un parámetro eficaz que me ayude en la toma de decisiones a la hora de enfocar el tratamiento y la prevención del proceso.
\end{abstract}

\section{PALABRAS CLAVE}

Patogenia, microbiología, mediadores de la inflamación, test genéticos, parámetros de destrucción periodontal, población de riesgo, acitvidad periodontal

Aceptado para publicación: Julio 2003

\section{INTRODUCCIÓN}

En el área de la salud las enfermedades adquieren trascendencia por dos motivos: por los daños que producen y por la prevalencia en la población general. La enfermedad periodontal es un proceso que no produce alteración vital de importancia, pero si la pérdida inexorable de la dentición en un período mayor o menor. Además, ésta viene acompañada de la pérdida del hueso alveolar que soporta los dientes. Esto conlleva que no se consigue una adecuada estabilidad de la prótesis y afecta a la capacidad de masticación, produciendo una manifiesta incapacidad. Actualmente, la única solución a éste problema es la utiliza- ción de implantes, lo que encarece y complica el tratamiento. El paciente pierde calidad de vida de una manera notoria, con el agravante de que la enfermedad suele afectar a pacientes de edad avanzada.

Por otra parte, la prevalencia en la población general es de la mas alta. Se calcula que en la población general el $40 \%$ de la población por encima de los 35 años tiene periodontitis moderada y entre el $4 \mathrm{y}$ el $8 \%$ periodontitis avanzada (1). Recientemente, se ha publicado un estudio epidemiológico de la salud oral en España, en el que constatan: una disminución de la caries, una mayor proporción de dentados en la población joven comparada con la adulta y un mante-

* Catedrático de Universidad. Facultad de Odontología. Universidad de Sevilla, 
nimiento de las cifras de prevalencia de la enfermedad periodontal (2). Además, si consideramos a los pacientes que acuden a la consulta, también se da en cifras parecidas. Por ello, la necesidad de tratamiento periodontal debe ser uno de los tratamientos mas demandados en nuestras consultas.

Para llevar a cabo un tratamiento apropiado es necesario, en primer lugar, contar con unos métodos de diagnóstico que nos lleven a conocer las características de cada enfermedad y poder realizar el tratamiento mas adecuado en cada paciente. Los métodos de diagnóstico de la enfermedad periodontal están influenciados por los conceptos que sobre la patogenia se tienen en ese momento. Por ello recordaremos los conceptos básicos que sobre la enfermedad se tiene actualmente, para repasar la utilidad de los métodos de diagnóstico periodontal con que contamos.

\section{PATOGENIA DE LA ENFERIMEDAD PERIODONTAL}

Actualmente, la enfermedad periodontal se considera que es un proceso de origen infeccioso que provoca una respuesta inflamatoria que en determinadas circunstancias provoca la reabsorción en altura del hueso alveolar y la consiguiente pérdida del diente.

El esquema más aceptado con respecto a la patogenia es el publicado por Page y Kornman (3). En esencia la periodontitis está iniciada y mantenida por un pequeño grupo de bacterias predominantemente gramnegativas, anaerobias o microaereófilas que colonizan el surco gingival. Pero se sabe que el concurso de las bacterias es insuficiente para provocar la enfermedad, hay otros factores involucrados. Las bacterias provocan la respuesta inflamatoria que a veces protege, pero otras es dañino para el propio organismo. Aparecen células inflamatorias como polimorfonucleares, linfocitos y macrófagos, y mediadores de la inflamación como citoquinas, prostaglandinas y metaloproteinasas y se producen anticuerpos ante los antígenos bacterianos que intentan controlar el proceso. Estos dos procesos: la infección y la inflamación, están influidos por distintos factores de riesgo. Un grupo que podemos considerar adquiridos y son: el tabaco, el estrés y enfermedades como la diabetes y otro grupo de tipo genético que modifican y alteran la respuesta. Estos producen una serie de fenómenos destructivos en el tejido conectivo y el hueso que provocan los signos clínicos de iniciación y progreso de la enfermedad.

Este esquema de patogenia no resuelve hechos clínicos como, el porqué en determinados pacientes el compo- nente inflamatorio se reduce a una gingivitis sin afectar al tejido de soporte y porqué en otras personas es capaz de destruirlo. Esto quedó reflejado hace años en el trabajo de Löe y cols (4) en el que demuestran en una población sin estar sometida a tratamiento periodontal a lo largo de los años como un grupo presentaba una evolución rápida, otro moderada y otro sin variación, teniendo todos unos índices de placa similares. Además, que el $30 \%$ de la población era responsable del $70 \%$ de la pérdida de inserción. El concepto que se deriva es el de población de riesgo. Este es importante ya que su identificación permitiría un tratamiento precoz y mas estrecho de ésta población.

Por otra parte la enfermedad periodontal es una enfermedad crónica cuya evolución se sabe actualmente que no es progresiva y continua. El modelo mas aceptado de evolución es por brotes al azar que afectan cada vez a distintos sitios (5), pero que ineludiblemente con el paso del tiempo producen la pérdida de la dentición. Existen fases de actividad y otras de inactividad en las que no se produce pérdida de soporte óseo (6). El conocer en que fase nos encontramos es importante para estar seguros de que el tratamiento es el responsable de la mejoría y no el paso a la inactividad. El objetivo del tratamiento tiene que ser el control de la enfermedad en el tiempo y no en un momento dado.

Por tanto los objetivos del diagnóstico de la enfermedad periodontal deben ser:

a) conocer el alcance del daño producido para en base a él realizar el tratamiento apropiado

b) identificar a la población de riesgo para iniciar un tratamiento precoz

c) controlar los períodos de actividad del proceso evitando la pérdida futura de soporte óseo

\section{DIAGNÓSTICO CLÍNICO DE LA ENFERIMEDAD PERIODONTAL}

Desde el punto de vista clínico existe una lesión ósea que se puede constatar mediante la comprobación de la profundidad del surco gingival y de la radiografía. Los dos métodos de diagnóstico clínicos apropiados son el sondaje periodontal y la radiología intraoral.

El sondaje se realiza mediante la sonda periodontal milimetrada que se introduce en el surco periodontal y mide la distancia con respecto a la línea amelocementaria. Se considera que la medida indicativa de salud es una pérdida de inserción máxima de 3 milímetros. La presión que se aplica no debe superar los 25 gr. En clínica se toma como referencia al peso de una carta, la presión con la que se sujeta la sonda es 
ligera de tal forma que pueda ser fácilmente quitada de la mano. Existen sondas de presión controlada electrónicamente que cuando se supera una presión suena una alarma, como la Viney Valley Research. En otras la presión se mide por la depresibilidad de un muelle, y en estas algunas pueden realizarse medidas registradas en el ordenador como la sonda de Florida que toma un punto de referencia como el borde incisal (7) o la sonda de Foster-Miller en que registra automaticamente la línea amelocementaria (8). El uso de estas sondas no suele estar muy extendido por ser mas complicadas en su uso, reduce el tacto del explorador y para el paciente es mas molesto, por tanto no aporta ventajas en el uso clínico diario reservándose su uso para la investigación clínica (9).

En la radiografía se debe apreciar que la cresta marginal se encuentra a unos 2 ó 3 milímetros de la línea amelocementaria. La técnica radiológica que se debe realizar es la técnica en paralelo, pues no superpone estructuras. En un paciente se realiza la serie periapical de radiografías con la que se obtiene información del estado general del proceso alveolar de la mandíbula y del maxilar (10).

El principal problema de estas dos técnicas es que constatan un hecho, la disminución de la altura del proceso alveolar, pero sin dar información de cuando se ha producido o si se está produciendo en ese momento. La única posibilidad de saber esto es de repetir al cabo del tiempo la exploración y constatar si los cambios se han producido o no. Pero debo de contar con la posibilidad de saber si el tratamiento es el correcto en el momento de su realización. Para ello, el único índice clínico que me predice de alguna manera el control del proceso periodontal es el sangrado al sondaje. En el trabajo de Lang y cols (11) demostraron que cuando el sangrado al sondaje se repite en un mismo sitio, tiene un valor predictivo positivo bajo, no es capaz de predecir adecuadamente una pérdida futura de inserción. En cambio, cuando es negativo si predice con mayor seguridad que no habrá una pérdida futura de inserción, tiene un alto valor predictivo negativo. No es un factor totalmente seguro, necesita de un tiempo para su correcta valoración y no identifica a la población de riesgo en los primeros momentos del proceso de destrucción.

También se han utilizado otros parámetros para medir cambios físicos o metabólicos como la temperatura subgingival y la aplicación de la medicina nuclear. Existe incluso comercializado una sonda de temperatura subgingival que mide los cambios por un cambio de color (12). En la medicina nuclear se ha utilizado el tecnecio 99 inyectado intravenosamente y explorando mediante un dispositivo miniaturizado que mide la radiación originada en el hueso alveolar viéndose incrementada en la pérdida ósea (13). Estas técnicas no aportan al sondaje y a la radiografía ninguna novedad por lo que no se suelen utilizar.

Como conclusión podemos decir que los métodos de diagnóstico clínico nos dan idea del daño producido, pero no identifican a la población de riesgo ni confirman si el tratamiento ha sido capaz de controlar los períodos de actividad periodontal. Para ello, se ha intentado durante todos estos años la búsqueda de un método de laboratorio capaz de suplir éstas deficiencias.

\section{DIAGNOSTICO DE LABORATORIO DE LA ENFERMEDAD PERIODONTAL}

La técnicas de laboratorio deben buscar el factor que produce esas diferencias, por lo tanto se pueden clasificar según lo que se pretende identificar:
A) Patógenos responsables
B) Mediadores de la respuesta inflamatoria
C) Parámetros de destrucción ósea y del coláge- no
D) Factores genéticos
E) Factores de riesgo ambiental y adquiridos

\section{A) Patógenos responsables}

La cavidad oral es una cavidad séptica, por definición, en la que se ha aislado mas de 300 especies bacterianas distintas. Para que sean capaces estas bacterias de permanecer en boca y producir patología es necesario que se depositen en forma de placa dental. La placa dental es un biofilm microbiológico que se define como "una población de bacterias englobadas en una matriz que se adhieren unas a otras o a distintas superficies". La identificación de la especie o grupo de especies bacterianas responsables de la enfermedad periodontal ha supuesto un enorme esfuerzo investigador en los últimos años. Se han identificado especies bacterianas asociadas con los distintos estadios de salud o enfermedad. Darveau (14) publicó recientemente una relación de los mismos. En esencia las bacterias asociadas con salud son eminentemente gram-positivas, estreptotococos y actinomices y sólo un 15\% bacilos gram-negativos, pero en la persona de edad avanzada se incrementa al 45\%. En la gingivitis se incrementa los gram-negativos hasta el 50\% y en la periodontitis un 70 a $80 \%$ son gram-negativos anaerobios. Las especies mas asociadas a destrucción periodontal son: Actinobacillus actinomycetemcomitans, Porphyromona gingivalis, Bacteroides forsythus, Prevotella intermedia, Fusobacterium nucleatum, Campylobacter rectus y Eikenella corrodens. 


\section{AVANCES}

Volumen 16 - No 1 - Abril 2004

La primera deducción de todo es la complejidad de la microflora y su variabilidad que hace que cambien los resultados entre distintos sitios de la cavidad oral, incluso dentro del mismo diente. Por lo tanto, la toma de muestra debe ser de la zona específica que nos interese estudiar.

En esencia los métodos de diagnóstico mas utilizados actualmente son:
a) cultivo
b) métodos de inmunodiagnóstico
d) sonda de ADN
e) la reacción en cadena de la polimerasa (PCR)
f) enzimología (15).

Durante algunos años se utilizaron las técnicas de microscopio de contraste de fases y de campo oscuro, en ellas se veían organismos móviles y espiroquetas sin identificar ninguna especie concreta mas, por ello no se suele utilizar actualmente por la poca información que ofrece.

a) El cultivo de las bacterias es el procedimiento clásico y que se toma como referencia para validar cualquier otro método nuevo. Es el único método válido para determinar la susceptibilidad antimicrobiana in vitro de los periodontopatógenos y es capaz de cuantificar todos los microorganismos viables de una muestra. El cultivo identifica sólo especies vivas a diferencia de los otros métodos. Sin embargo como factor negativo necesita equipo sofisticado, personal muy entrenado, lleva tiempo y es costoso. La técnica consiste en la toma de una muestra de placa subgingival, previo secado con aire de la superficie del diente y eliminación de la placa supragingival, mediante una punta de cureta cortada o con una punta de papel absorbente. Se introduce en un medio de transporte con CO2 y se remite al laboratorio lo antes posible. Se homogeneiza la muestra y se cultiva en medio anaeróbico en placa con agar y suplementado con distintos productos. Los medios no selectivos cuantifican las colonias que se desarrollan. Los medios selectivos emplean distintas sustancias que impiden el crecimiento de determinadas especies y facilitan otras. La identificación de las colonias se realiza por la morfología y otras características conocidas.

b) Los métodos de inmunodiagnóstico emplean anticuerpos específicos contra antígenos bacterianos, que se hacen objetivables mediante distintas técnicas. El método no requiere bacterias viables y no es afectado mucho por la técnica de recogida de muestra. En cambio, la fiabilidad depende mucho de los reactivos utilizados. Los límites de detección son peores que en la sonda $A D N$ y la PCR. Proveen una estimación cuantitativa o semicuantitativa de los gérmenes. Se ha utilizado una técnica de inmunofluorescen- cia para la detección del A actinomycetemcomitans, una citometría de flujo basada en la unión de anticuerpos monoclonales específicos a lipopolisacáridos para la $\mathrm{P}$ gingivalis y una prueba de inmunoensayo asociado a enzimas.

c) La sonda de ADN utiliza segmentos de ADN cargados con un enzima o un radioisótopo complementarios del correspondiente del gérmen diana. Cuando se utiliza toda la secuencia de $\mathrm{ADN}$ puede tener reacción cruzada con otros gérmenes, es necesario contar con secuencias de oligonucleótidos específicos de especie que eviten esto (16).

d) La PCR se basa en una amplificación repetida de una región de $\mathrm{ADN}$ flanqueada por un par específico de la especie diana. La presencia de la ampliación indica la presencia del microorganismo. Entre todos los métodos es el que tiene un límite de detección menor, pues identifica cuando hay de 3 a 5 bacterias y no muestra reacciones cruzadas con otros elementos. Casi todas las técnicas de PCR determinan la presencia o ausencia de determinados microorganismos sin cuantificar. Algunas técnicas han añadido productos a la cadena que mediante cambios colorimétricos pueden llegar a cuantificar. Esta técnica es la ideal para los microorganismos periodontopatógenos, pero no suelen dar resultados cuantitativos y son positivos ante tan bajo número de gérmenes que puede que no tenga significación clínica (17).

e) En la enzimología incluimos las distintas técnicas que detectan un patógeno identificando la presencia de una enzima específica de una o más especies bacterianas. En éste grupo se encuentra el BANA (Perioscan), comercializado en nuestro país y basado en la presencia de una tripsina específica de $P$. Gingivalis, B. Forsythus y T. dentícola. Se toma la muestra de placa subgingival con cureta, se coloca en una cartulina, se incuba a $55^{\circ}$ durante 15 minutos y se lee según el cambio de color.

En estos últimos años se ha abierto una nueva perspectiva en el estudio de los patógenos periodontales y es el papel que en la etiología pueden cumplir los virus, concretamente el grupo de los virus herpes. Esto puede abrir en el futuro otras posibilidades de diagnóstico en el laboratorio. Las técnicas (18) que en este campo se han utilizado para establecer la relación son:

- la descripción con microscopio electrónico de cuerpos intranucleares y partículas parecidas a virus en las biopsias de encía

- técnica de la PCR para herpes en sangre, saliva, bolsa periodontal y en las células inflamatorias de la biopsia de encía 
Como final es necesario destacar que aunque en bolsas profundas activas se aíslen determinados gérmenes no sabemos si son los responsables de la enfermedad o son comensales, se aprovechan de las condiciones locales de crecimiento para proliferar. Se ha comprobado que a veces la presencia de las bacterias y virus considerados periodontopatógenos pueden aislarse en individuos sanos. Un mejor entendimiento de la patogenia y de los mecanismos de repuesta deben aclarar todos estos aspectos. Hoy en día los procedimientos de diagnóstico microbiológicos se utilizan en la clínica en casos de periodontitis que no responden al tratamiento y para comprobar la eliminación de algún periodontopatógeno específico.

\section{B) Mediadores de la respuesta inflamatoria}

Estos patógenos van a provocar una respuesta inflamatoria de defensa ante esa agresión en el paciente. La hipótesis más probable que hoy se admite es que exista algún factor a este nivel que explique porqué en determinados pacientes se produce destrucción periodontal y en otros no, con la misma microflora. Es el concepto, ya referido previamente de susceptibilidad y población de riesgo.

Fundamentalmente, todas las técnicas van dirigidas a buscar los mediadores de la inflamación en dos niveles: a)local, b) general.

a) A nivel local tenemos dos fuentes: la saliva y el fluido crevicular. La saliva como fluido protector de toda la cavidad oral indudablemente tiene que influir en la capacidad de crecimiento bacteriano. Las técnicas de recogida de muestra son sencillas. Normalmente en periodoncia se utiliza la saliva mixta, estimulada o no con cera para conseguir una adecuada cantidad. Debe hacerse siempre a la misma hora y en las mismas condiciones de ayuno o ingesta, suele realizarse en ayunas por la mañana. No suele utilizarse la recogida de saliva parotídea ni submaxilar mediante la canalización de los conductos excretores, pues ésta saliva se segrega en gran cantidad en la estimulación de las comidas. La saliva de las glándulas menores es la que se segrega en los intervalos entre comidas y tiene mayor trascendencia para la homeostasia de la cavidad oral (19). En una revisión reciente (20) se analiza los factores que se han utilizado para el diagnóstico periodontal en la saliva, concluyendo que todavía se requieren estudios longitudinales apropiados para aconsejar su utilización.

El fluido crevicular es producido en el surco gingival como consecuencia de la extravasación de plasma que se produce en la inflamación. Por tanto, su sola presencia indica una actividad inflamatoria. Además, es posible estudiar en él distintos componentes inflamatorios. Para ello se utiliza papel absorbente corta- do en tiras finas y colocados en el surco gingival. El surco elegido para la toma de muestra previamente se ha secado con aire, se ha aislado para que la saliva no lo contamine y se ha eliminado placa supragingival. Posteriormente, utilizando una determinada cantidad de papel y mediante técnicas bioquímicas puede cuantificarse la cantidad de distintas sustancias presentes.

El Periotron es un aparato electrónico que mide la cantidad de fluido basado en la cantidad de papel humedecido, pero existe una gran variabilidad en los resultados lo que hace a Deinzer y cols poner en duda la validez de los resultados volumétricos (21).

Se han utilizado otras técnicas de recogida de fluido, por ejemplo mediante micropipetas introducidas en el surco, pero se dejaron de utilizar por distintos problemas como la viscosidad excesiva de la saliva en determinadas circunstancias (22); o mediante la introducción de un buffer y su recogida, pero sólo se ha utilizado en investigación (23).

b) A nivel general, puede recogerse una muestra de sangre. En ella se pueden valorar los elementos formes, su número y funcionalidad como la capacidad de defensa de los neutrófilos, y en el suero cuantificar los distintos productos de la respuesta inmune.

Los distintos mediadores de la inflamación que se han utilizado en periodoncia se podrían dividir en tres grupos:

a) los producidos en la respuesta inmune,

b) los mensajeros intercelulares que sirven de señal entre las células

c) las enzímas líticas que aparecen en el proceso inflamatorio.

d) radicales libres

a) La respuesta inmune se produce a dos niveles: celular y humoral. A nivel celular se busca el número y la funcionalidad de las células que intervienen en la inflamación. Los distintos estudios se han circunscrito sobretodo a dos grupos: los neutrófilos y los monocitos. En los neutrófilos se ha estudiado la quimiotaxis, la capacidad fagocítica y la producción de radicales superóxido (24). En los monocitos se ha medido la mayor respuesta ante los lipopolisacáridos (prostaglandina E2) pensando en que el paciente susceptible presenta una respuesta mayor ante los gérmenes gram- (25).

A nivel humoral se ha medido la producción de anticuerpos ante bacterias periodontopatógenas. Un número elevado de trabajos han relacionado un nivel mayor de anticuerpos con una severidad mayor de la periodontitis. Lo importante sería correlacionar esa 
elevación con fases de actividad periodontal. No se sabe si la elevación es una protección ante un agente determinado y por tanto, inactividad, o si es indicativo de actividad. Se ha podido medir el nivel de anticuerpos a nivel del fluido crevicular y en plasma pero los resultados no son definitorios. Donde existe una mayor correlación entre la disminución de anticuerpos y la severidad de la enfermedad se da en las periodontitis de aparición temprana pero no en la forma crónica del adulto (26). En un trabajo de Craig et al (27) encuentran una correlación entre los valores de IgG de la $P$. gingivalis y la destrucción periodontal, pero también encuentran diferencias en los valores atribuidos a factores étnicos, raciales y socioeconómicos.

b) Los mensajeros intercelulares son las también denominadas citoquinas, es un grupo de péptidos producidos por el hospedador cuya misión es transmitir ordenes entre distintas células, promoviendo o inhibiendo la secreción de sustancias o de procesos bioquímicos. En el fluido crevicular se ha encontrado IL-lalfa y beta en concentraciones superiores en la periodontitis que en la gingivitis y en la salud (28), mientras que el tratamiento con el raspado no las modifica y el tratamiento con cirugía las eleva (29). En un trabajo reciente de Engebretson (30) comprueban que la IL-1beta se encuentra en valores superiores en la periodontitis severa, pero en éstas no hay diferencias en lo obtenido en zonas con pérdida de inserción $<4 \mathrm{~mm}$ comparadas con zonas $>4 \mathrm{~mm}$. La IL-6 se incrementa en la periodontitis refractaria (31). La inhibición del factor de necrosis tumoral (TNalfa) impide la pérdida de hueso en la periodontitis experimental (32).

c) Las enzimas líticas son un conjunto de sustancias segregadas por distintas células que desorganizan y destruyen el tejido conectivo y óseo. Podríamos agruparlas en tres grupos: las derivadas del ácido araquidónico, las metaloproteinasas y otras.

Entre las derivadas del ácido araquidónico, sustancia derivada de los fosfolípidos de la membrana celular por la vía de la ciclooxigenasa, se encuentra la prostaglandina E2. Produce un grado elevado de reabsorción ósea tanto in vitro como in vivo. Se ha demostrado que se encuentra elevada en la periodontitis, aunque se observan grandes variaciones entre individuos y entre distintas zonas de la cavidad oral (33). En un estudio longitudinal se ha demostrado que los pacientes con actividad periodontal y pérdida de inserción periodontal, se encuentra muy elevada las cifras de prostaglandina E2 en el fluido crevicular (34). Preshaw y Heasman demuestran en pacientes periodontales sin tratamiento que existe un incremento en las cifras durante los tres meses que controlan a los pacientes (35).
Las metaloproteinasas son un grupo de endopeptidasas producidas por células inflamatorias como los neutrófilos y los macrófagos, y por las propias del periodonto como los fibroblastos, los osteoblastos y las células epiteliales. Son enzimas destructoras del tejido conectivo, involucradas en innumerables procesos de remodelación embrionaria y patológicos como artritis y cáncer. Entre ellas la mas estudiada ha sido la colagenasa. Se ha visto que está elevada en el fluido crevicular en los sitios con pérdida de inserción previa explorada clínicamente o con radiografías (36). Incluso se ha comercializado un test para la clínica basado en la colagenasa del fluido crevicular. Se obtiene mediante tiras de papel muestra del fluido crevicular, se incuba a $43^{\circ}$ en gel y se torna en color azul el positivo (37). Otras proteasas como las catepsinas, la elastasa, tripsina, quimotripsina y glicilpropil dipeptidasa se han visto que se incrementan en bolsas profundas y pérdidas de inserción severas, disminuyendo con el tratamiento (38). También, se ha elaborado un test para medir la elastasa mediante fluorescencia, viéndose su relación con los sitios con pérdida de inserción (39). Soell et al (40) han demostrado que la destrucción tisular resulta de un desequilibrio entre las metaloproteinasas y sus inhibidores tisulares.

Dentro del grupo de otras enzímas líticas incluimos dos: la beta glucoronidasa, enzima lisosómica liberada por los polimorfonucleares, y la aspartatoaminotransferasa, enzima intracelular liberada en la necrosis, las cuales se han relacionado con destrucción periodontal $(41,42)$. De la aspartatoaminotransferasa existe un test comercializado (PocketWatch).

d) Uno de los factores que recientemente se ha analizado es el papel que los radicales libres y las especies reactivas del oxígeno tienen en la enfermedad periodontal, ya que se les ha atribuido algún papel en enfermedades como la arterioesclerosis, algunas enfermedades neurológicas, el cáncer y el envejecimiento (43). Entre éstos el mejor estudiado ha sido el $\mathrm{NO}$, molécula involucrada en innumerables procesos fisiológicos e inflamatorios. Se ha demostrado que existe un incremento de la óxido nítrico sintetasa, enzima productora de NO, en un modelo de periodontitis en ratas (44) y que la flora de la placa bacteriana induce un incremento en las especies reactivas del nitrógeno (45).

\section{C) Parámetros de destrucción ósea y del colágeno}

El producto de la inflamación generada por la infección es una destrucción del soporte periodontal que afecta al tejido conectivo y al hueso. Esto se puede objetivar mediante: a) técnicas histológicas

b) análisis bioquímico del fluido crevicular. 
a) En las técnicas histológicas se toma una biopsia mediante un punch o bisturí circular o un fragmento de encía mediante incisiones paralelas, siempre procurando regenerar el tejido extraído en su totalidad. En ellas se pretende examinar mediante el microscopio algún componente inflamatorio distintivo del proceso de destrucción. El estudio se realiza con microscopía óptica o electrónica. El fijador utilizado para el óptico es formol tamponado al 10\% y para el electrónico glutaraldehido al $2 \%$.

En el microscopio óptico las técnicas van dirigidas a identificar alguna modificación de la celularidad inflamatoria y algún cambio en el metabolismo del colágeno midiendo la cantidad de fibras colágenas o grado de apoptosis (46).

Existen dos tipos de técnicas las coloraciones clásicas basadas en las diferencias bioquímicas de los tejidos que los colorea de distinta forma y las técnicas de inmunoperoxidasa en las que la coloración se basa en la reacción antígeno anticuerpo. Estas últimas han adquirido una gran predominancia en los últimos años porque nos permiten identificar distintas sustancias indicativas del estado del tejido con una gran precisión.

Los colorantes más utilizados son: la hematoxilina eosina que es la técnica general que nos permite ver las distintas estructuras de los tejidos y para identificar las distintas fibras del tejido conectivo están las técnicas tricrómico de Masson, Van Gieson, Picro Mallory y Verhoeff.

Dentro de las técnicas de inmunoperoxidasa tenemos:

Mib: mide la actividad proliferativa del epitelio y del estroma

VegF: mide la vascularización

CD34: mide vascularización células plasmáticas

P53 y bcl2: mide la angiogénesis y la apoptosis

CDla: identifica las células de Langerhans

TgF: tiñe los fibroblastos y el endotelio activado

CD68: tiñe los macrófagos

CD4: tiñe los linfocitos T-helper

CD8: tiñe los linfocitos T-citotóxicos

CD20: tiñe linfocitos B

Actina: identifica las fibras de músculo liso

Las técnicas de microscopía electrónica nos permiten identificar alteraciones intracelulares sobretodo de los fibroblastos con cambios en su estructura por la aparición de fibras de músculo liso o del estado de las mitocondrias que nos indica el estado metabólico.

b) El análisis del fluido crevicular puede determinar el nivel de varios productos de destrucción tisular como la hidroxiprolina, fibronectina, glicosaminoglicanos, calprotectina y fosfatasa alcalina. Por ejemplo ésta última mide la función de los osteoblastos y de los neutrófilos. Se elaboró en primer lugar un test por quimioluminiscencia tomando muestra del fluido crevicular con tiras de papel y encontrando valores elevados en la periodontitis (47), luego ha sido mejorado con un test colorimétrico, PeriogardTM, el cual pretende distinguir entre sitios sanos y enfermos (48). Pero, como señala Lamster en su revisión (49) aunque sirven para el diagnóstico de la destrucción periodontal, no es seguro que determinen la probabilidad de desarrollar una periodontitis a partir de una gingivitis. Todas éstas técnicas no tienen actualmente indicación clínica usándose sólo en el campo de la investigación. También se puede analizar la osteocalcina que es una de las proteínas de la matriz ósea, pero los resultados por ahora no son prometedores (50). No obstante como señala Giannobile (51) el desarrollo de alguna prueba que determine el grado de destrucción ósea debe seguir siendo buscado.

\section{D) Factores genéticos}

Uno de los aspectos de la periodontitis estudiado en los últimos años es su carácter hereditario (52). Se sabe que en determinadas familias existe una mayor prevalencia de la enfermedad, sobretodo en las periodontitis de aparición temprana, donde se habla de que pueda existir una herencia autosómica dominante (53). La búsqueda del gen responsable de la alteración patológica ha sido estudiado intensamente, cualquiera de los genes que regulen la producción de los factores de la inflamación que hemos descrito, podría estar involucrado (54). Recientemente se ha elaborado un test genético (PSTTM) para evaluar el riesgo periodontal. El test determina la posesión de una combinación de alelos en dos genes de la IL-1 (55). Los resultados de estudios realizados son controvertidos, en unos se relaciona con destrucción periodontal (56) y en otros no (57), por lo que aunque ésta área deba de tener un desarrollo en el futuro, todavía no existen pruebas útiles desde el punto de vista clínico. En una reciente revisión de Greenstein y Hart (58) concluyen que los clínicos deben ser cautos para sacar conclusiones sobre los test que están comercializados que buscan genes de la IL-1. También, se ha elaborado un test para medir el gen del TNF alfa sin poder relacionarlo con la susceptibilidad ni severidad periodontal (59).

\section{E) Factores de riesgo ambiental y adquiridos}

En este grupo incluimos el análisis de todos los factores de riesgo que influyen en el desarrollo del proceso de destrucción periodontal. El principal factor de riesgo ambiental reconocido es el tabaco debido a la alteración de la microvascularización, microflora e inmunoglobulinas principalmente (60). Dentro de las 
enfermedades relacionadas con la periodontitis se encuentran todas que de alguna manera reducen la protección de los neutrófilos como: agranulocitosis, neutropenia cíclica, síndrome de Chediak Higashi, síndrome de Down, síndrome de Papillon-Lefevre entre otras. Destacan de una manera especial la diabetes, la enfermedad cardiovascular y la osteoporosis. La diabetes se asocia con una mayor prevalencia y mayor severidad de periodontitis (61) y la enfermedad cardiovascular se ha relacionado epidemiológicamente con la periodontitis (62). Es preciso contar con un mejor conocimiento del estado de salud general de estos pacientes para un adecuado control del proceso periodontal. Se ha relacionado la pérdida de dientes con la osteoporosis (63) y se ha comprobado que la osteopenia y osteoporosis son indicadores de riesgo para la periodontitis (64).

El diagnóstico adecuado de estos procesos debe permitir un mejor control de la enfermedad periodontal, para ello es preciso utilizar los métodos diagnósticos precisos para evaluar el alcance de la situación de riesgo.

\section{DIAGNÓSTICO DE PERIIMPLANTITIS}

Los implantes son una de las terapéuticas mas utilizadas hoy en día y que viene a resolver los problemas que la pérdida dentaria produce para la estabilidad de la prótesis. La incógnita mas importante que plantea es si los tejidos blandos que rodean el implante son capaces de mantener la salud periodontal. Hemos de tener en cuenta que el periodonto se produce por un proceso de desarrollo en la erupción dentaria, en cambio la mucosa periimplantaria se produce a través de un mecanismo de cicatrización. Hoy en día se admite que la estructura de la mucosa peirimplantaria es parecida a la encía, cambia la dirección de las fibras colágenas que son paralelas al implante, pero el resto desde el punto de vista morfológico y funcional es exactamente igual (65). Por otra parte, se sabe que ese surco periimplantario puede ser reservorio para los microorganismos patógenos desde el punto de vista periodontal.

Los implantes pueden fracasar en un proceso que se ha venido en denominar periimplantitis, su etiología se atribuye o a una sobrecarga o a una infección parecida a la periodontitis. Por ello todos los conceptos que hemos venido desarrollando para el diagnóstico de la periodontitis sirven para la periimplantitis.

\section{CONCLUSIÓN}

Para hablar de un test diagnóstico fiable es preciso hablar de sensibilidad, especificidad, valor predictivo positivo y valor predictivo negativo. Para ello, cuando analizamos los resultados de un test podemos encontrar que es positivo estando o no la enfermedad presente y que es negativo estando o no presente la enfermedad. Se define como sensibilidad como la probabilidad de que un resultado sea positivo cuando la enfermedad está presente, no hay ningún positivo estando la enfermedad ausente. La especificidad es la probabilidad de que el test sea negativo y la enfermedad esté ausente, el test no da como negativo con la presencia de la enfermedad. El valor predictivo positivo es la probabilidad de que la enfermedad esté presente cuando el test es positivo. El valor predictivo negativo es la probabilidad de que la enfermedad esté ausente cuando el test es negativo. Lo ideal es que todos los test tengan un valor predictivo de 1,00. Esto es imposible en Ciencias de la Salud, se considera aceptable un valor de 0,7. Pero los valores aceptables dependen de los riesgos que se puedan asumir con los errores. Así, una enfermedad mortal y que pueda ser tratada adecuadamente, se le debe pedir al test una sensibilidad máxima. El error es de fatales consecuencias y es secundario la especificidad.

En periodoncia como hemos visto contamos con un test clínico, el sondaje periodontal, adecuado para evaluar la presencia o ausencia de enfermedad, pero no para predecir el desarrollo futuro del proceso o para identificar a la población de riesgo antes de la aparición de la enfermedad. Para evaluar la capacidad de predicción de un test se utiliza el odds ratio que se define como la medida del riesgo de desarrollar la enfermedad. También se utiliza el concepto de riesgo relativo como la posibilidad de desarrollar la enfermedad cuando se expone a un factor de riesgo. Hemos visto que ningún test de diagnóstico de laboratorio es capaz de darnos una capacidad de predicción adecuada, quizás en gran medida debido a las enormes lagunas que la patogenia de la enfermedad periodontal tiene para nosotros. No obstante, es un campo en el cual debe seguir investigándose para conseguir en el futuro el diagnóstico preciso del proceso en todas sus vertientes.

\section{ABSTRACT}

The periodontal disease is very important in the general health status of our patients. We need an adequate diagnosis of the process to treat and prevent successfully. The diagnosis depends on the concept about the illness is generally accepted nowadays. We revise all the diagnostic procedures we can use in the clinic. We conclude that the clinic diagnostic method with the periodontal probe is the best way to know the des- 
tructive bone process and treat it. The others methods that study the bacteria, inflammatory mediators, parameters of bone and collagen resorption, genetic tests cannot identify the high-risk patients and the period of activity. The reason could be the lack of a completely knowledge of the pathogenic process involved in the periodontal disease. We have to work in this field in the future to improve the treatment and prevention of the periodontitis.

\section{KEY WORDS}

Pathogenia, microbiology, inflammatory mediators, genetic test, periodontal destruction parameters, high risk patient, periodontal activity

\section{CORRESPONDENCIA}

\author{
Facultad de Odontología \\ Universidad de Sevilla \\ $\mathrm{C} /$ Avicena s/n \\ 41009 Sevilla \\ tfno 954481120 \\ email: pbullon@us.es
}

\section{BIBLIOGRAFÍA}

1. Gonzalez V, P. Bullón. Estudio Epidemiológico piloto de salud oral y necesidades de tratamiento en un grupo de adultos de 35-44 años en Sevilla. Archivos de Odontoestomatología preventiva y Comunitaria 1989 1:25-31.

2. Llodra-Calvo JC, Bravo-Pérez M, Cortés-Marticorena Fj. Encuesta de salud oral en España (2000) RCOE 2002; 7: 19-63.

3. Page RC, Kornman KS. The pathogenesis of human periodontitis: an introduction. Periodontol 2000 1997; 14: 9-11.

4. Loe H, Anerud A, Boysen H, Morrison E. Natural history of periodontal disease in man. Rapid, moderate and no loss of attachment in Sri Lankan laborers 14 to 46 years of age. J Clin Periodontol 1986; 13: 431-45.

5. Socransky S, Haffajee AD, Goodson JM, Lindhe J. New concepts of destructive periodontal disease. J Clin Periodontol 1984; 11:21-32.

6. Bullon P, Rios JV, Machuca G, Martinez-Sahuquillo A, Velasco E. Susceptibilidad y actividad periodontal: conceptos actuales. Periodoncia 1993 3: 78-88.

7. Gibbs CH, Hirschfeld JW, Lee JG, Low SB, Magnusson I, Yernen P, Clark HB. Descrption and clinical evaluation of a new computerized periodontal probe - The Florida Probe. J Clin Perioodntol 1988; 15: 137-44.

8. Jeffcoat MK, Jeffcoat RL, Jens SC, Captain K. A new periodontal probe with automated cemento-enamel junction detection. J Clin Periodontol 1986; 13: 276-80.

9. Armitage GC. Clinical evaluation of periodontal disease. Periodontology 2000 1995; 7: 39-53.

10. Jeffcoat MK, Chung-Wang I, Reddy MS. Radiographic diagnosis in periodontics. Periodntol 2000 1995; 7: 54-68.

11. Lang NP, Joss A, Orsanic T, Gusberti FA, Siegrist BE. Bleeding on probing. A predictor for the progression of periodontal disease?. J Clin Periodontol 1986 13: 590-6.

12. Haffajee AD, Socransky SS, Goodson JM. Subgingival temperature (II). Relation to future attachment loss. J Clin Periodontol 1992; 19: 409-16.

13. Jeffcoat MK et al. Detection of active alveolar bone destruction in human periodontal disease by analysis of radiopharmaceutical uptake after a single injection of 99mTc-methylene diphophonate. J Periodont Res 1986; 21:677-84.

14. Darveau RP, Tanner A, Page R. The microbial challenge in periodontitis. Periodontol 2000 1997; 14: 12-32.

15. Chen C, Slots J. Microbiological tests for Actinobacillus actinomytemcomitans and Porphyromona gingivalis. Periodontology 2000 1999; 20: 53-64.

16. Zambon JJ, Haraszthy VI. The laboratory diagnosis of periodotnal infections. Periodontol 2000 1995; 7: 69-82.

17. Chen C, Slots J. Microbiological tests for Actinobacillus actinomycetemcomitans and Porphyromona gingivalis. Periodontol 2000 1999; 20: 53-64.

18. Contreras A, Slots J. Herpesvirus in human periodontal disease J Periodontol Res 2000; 35: 3-16.

19. Bullon P. Fisiopatologia de las glandulas salivales. En: Medicina Oral Eds Bagan JV, Ceballos A, Bermejo, Aguirre JM, Peñarrocha M. Editorial: MASSON S.A. pag 257-264 Barcelona 1995.

20. Kaufman E, Lamster IB. Analysis of saliva for periodotnal diagnosis. A review. J Clin Periodontol 2000; 27: 453-465.

21. Deinzer R, Mossanen BS, Herfoth A. Methodological considerations inb the assessment of gingival crevicular fluid volume. J Clin Periodontol 2000; 27:481-8.

22. Cimasoni G. Crevicular fluid updated. En: Myers HM ed. Monographs in Oral science. Basel: S Karger, 1983.

23. Skaspi H, Lehner T. A crevicular washing method for investigating immune components of crevicular fluid in man. J Periodont Res 1976;11: 19-24.

24. Bullon P, Pascual A, Fernandez-Novoa MC, Borobio MV, Muniain MA, Camacho F Late onset papillon-lefevre syndrome? A chromosomic, neutrophil function and microbiological study.J Clin Periodontol 1993 20: 662-7.

25. Garrison SW, Nichols FC. LPS-elicited secretory responses in moncytes: Altered release of PGE2 but not 1L-1 beta in patients with adult periodontitis. J Periodontol Res 1989; 24: 88-95. 
26. Lamster IB, Grbic JT. Diagnosis of periodontal disease based on analysis of the host response. Periodontol 2000 1995; 7: 83-99.

27. Craig RG et al. Serum IgG antibody response to periodontal pathogens in minority populations: relationship to periodontal disease status and progression. J Clin Periodontol 2002; 37: 132-46.

28. Massada MP, Persson R, Kenny JS, Lee SW, Page RC, Allison AC. Measurement of interleukin- 1 alfa and 1 beta in gingival crevicular fluid: Implications for the pathogenesis of perioodntal disease. J Periodont Res 1990; 25: 156-63.

29. Reinhardt RA et al. IL-1 in gingival crevicular fluid following closed root planing and papillary flap debridement. J Clin Periodontol 1993; 20: 514-9.

30. Engebretson SP, Grbic JT, Singer R, Lamster IB. GCF ILlbeta profiles in periodontal disease. J Clin Peruiodontol 2002; 29: 48-53.

31. Reinhardt RA et al. Gingival fluid IL-1 and IL6 in refractory periodontitis. J Clin Periodontol 1993; 20: 225-31.

32. Graves DT et al. Interleukin-1 and tumor necrosis factor antagonists inhibit the progression of inflammatory cell infiltration toward alveolar bone in experimental periodontitis. J Periodontol 1998; 69: 1419-25.

33. Offenbacher S, Farr DH, Goodson JM. Measurement of prostaglandin E in crevicular fluid. J Clin Periodotol 1981; 8: 359-67.

34. Offenbacher S, Odle BM, Van Dyke TE. The use of crevicular fluid prostaglandin E2 levels as a predictor of periodontal attachment loss. J Periodont Res 1986; 21: 101-12.

35. Preshaw PM, Heasman PA. Prostaglancin E2 concentrations in gingival crevicular fluid: observations in untreated chronic periodontitis. J Clin Periodontol 2002; 29: 15-20.

36. Villela B, Cogen RB, Bartolucci AA, Birkedal-Hansen H. Collagenolitic activity in crevicular fluid from patients with chronic adult periodontitis, localized juvenile periodontitis and gingivitis, and from healthy control subjects. J Periodotnol Res 1987; 22: 381-9.

37. Bowers JE, Zarhadnik RT. An evaluation of a chairsideprotease test for use in periodontal diagnosis. J Clin Dentist 1989; 1: 106-9.

38. Cox SW, Eley BM. Cathepsyn B/L-, elastase-, tryptase-, and dipeptidyl peptidase IV-like activities in gingival crevicular fluid. A comparison of levels before and after basic periodontal treatment of chronic periodonttis patients. J Clin Periodontol 1992; 19: 333-9.

39. Palcanis $\mathrm{KG}$ et al. Elastase as an indicator of periodotnal disease progression. J Periodontol 1992; 63: 237-42.

40. Soell M, Elkaim R, Tenenbaum H. Cathepsin C, matriz metalloproteinases, and their tissue inhibitors in gingiva and gingival crevicular fluid from periodontitis-affected patients. J Dent Res 2002; 81: 174-8.
41. Lamster IB et al. Lysosomal and cytoplasmic enzyme activity, crevicular fluid volume, and clinical parameters characterizing gingival sites with shallow to intermediate probing depths. J Periodontol 1987; 58: 614-21.

42. Imrey PB et al. A cross sectional analysis of aspartate aminotransferase in human gingival crevicular fluid. J Periodntol Res 1991; 26: 75-84.

43. Battino AM, Bullon P, Wilson M, Newman H. Oxidative injury and inflammatory periodontal diseases: the challenge of anti-oxidants to free radicals and reactive oxygen species. Crit Rev Oral Biol Med 1999; 10: 458-76.

44. Lohinai Z, Benedek P, Feher E, Gyorfi A, Rosivall L, Fazekas A, Salzman AL, Szabo C. Protective effects of mercaptoethylguanidine, a selective inhibitor of inducible nitric oxide synthase, in ligature-induced periodontitis in the rat. Br J Pharmacol. 1998; 123: 353-60.

45. Lohinai Z, Stachlewitz R, Virag L, Szekely AD, Hasko G, Szabo C. Evidence for reactive nitrogen species formation in the gingivomucosal tissue. J Dent Res. 2001; 80: 470-5.

46. Zappa U. Histology of the periodontal lesion: implications for diagnosis. Periodontol 2000 1995; 7: 22-38.

47. Chapple ILC et al. A new ultrasensitive chemoluminiscent assay for the site-specific quantification of alkaline phosphatase in gingival crevicular fluid. J Periodont Res 1993; 28: 266-73.

48. Magnusson I et al. A multicenter clincial trial of a new chairside test in distinguishing between diseased and healthy sites (II). Association between site type and test outcome before and after therapy. J Periodontol 1996; 67: 589-96.

49. Lamster IB. Evaluation of components of gingival crevicular fluid as diagnostic tests. Ann Periodontol 1997; 2: 123-37.

50. Lee AJ, Walsh TF, Hodges SJ, Rawlinson A. Gingival crevicular fluid osteocalcin in adult periodontitis. J Clin Periodontol 1999; 26: 252-6.

51. Giannobile WV. Crevicular fluid biomarkers of oral bone loss. Curr Opin Periodontol 1997;4:11-20.

52. Hassell TM, Harris EL. Genetic influences in caries and periodontal diseases. Crit Rev Oral Biol Med 1995; 6: 319-42.

53. Mazarita ML et al. Evidence for autosomal dominant inheritance and race specific heterogeneity in earlyonset periodontitis. J Periodontol 1994; 65: 623-30.

54. Hart HC, Korman KS. Geentic factors in the pathogenesis of periodontitis. Periodontol 2000; 14: 202-15.

55. Kornman KS et al. The interleukin-l genotype as a severity factor in adult periodontal disease. J Clin Periodontol 1997; $24: 72-7$.

56. Gore EA et al. Interleukin-1beta+3953 allele 2: association with disease status in adult periodontitis. J Clin Periodontol 1998; 25: 781-5. 
57. McGuire MK, Nunn ME. Prognosis versus actual outcome. IV. The effetctiveness of clinical parameters and IL-1 genotype in accurately predicting prognoses and tooth survival. J Periodontol 1999; 70: 49-56.

58. Greenstein G, Hart TC. A critical assessment of interleukin-l (IL-1) genotyping when used in a genetic susceptibility test for severe chronic periodontitis. J Periodontol 2002; 73: 231-47.

59. Craandijk J et al. Tumor necrosis factor-alfa gene polymorphisms in relation to perioodntitis, J Clin Periodontol 2002; 29: 28-34.

60. Machuca G, Rosales M, Lacalle JR, Machuca C, Bullon P. Effect of cigarette smoking on periodontal health status of healthy young adults. J Periodontol 2000; 71:73-8.

61. Mealey BL. Diabetes and periodontal disease: two sides of a coin. Compend Contin Educ Dent. 2000; 21: 943-6.

62. Abou-Raya S, Naeem A, Abou-El KH, El BS. Coronary artery disease and periodontal disease: is there a link? Angiology. 2002; 53: 141-8.

63. Krall EA, Garcia RI, Dawson-Hughes B. Increased risk of tooth loss is related to bone loss at the whole body, hip, and spine. Calcif Tissue Int 1996; 59: 433-7.

64. Tezal $\mathrm{M}$ et al. The relationship between bone mineral density and periodontitis in postmenopausal women. J Periodontol 2000; 71: 1492-8.

65. Bullon P. Marginal tissues integration at different implants. En: Proceedings of the 3rd European Workshop on periodontology. Lang NP, Karring T, Lindhe J. Pag 175184. Quintessence. Berlín 1999. 